\title{
An Intelligent Gripper Design for Autonomous Aerial Transport with Passive Magnetic Grasping and Dual-Impulsive Release
}

\author{
Usman A. Fiaz ${ }^{1}$, M. Abdelkader ${ }^{2}$, and Jeff S. Shamma ${ }^{2}$
}

\begin{abstract}
We present a novel gripper design for autonomous aerial transport of ferrous objects with unmanned aerial vehicles (UAVs). The proposed design uses permanent magnets for grasping, and a novel dual-impulsive release mechanism, for achieving drop. The gripper can simultaneously lift up to four objects of arbitrary shape, in fully autonomous mode, with a $100 \%$ rate of successful drops. We optimize the system subject to realistic constraints, such as the simplicity of design and its sturdiness to aerial maneuvers, payload limits for multirotor UAVs, reliability of autonomous grasping irrespective of the environment of operation, active power consumption of the gripper, and its comparison with the existing technologies. We describe the design concepts, and the hardware, and perform extensive experiments in both indoor and outdoor environments, with two multi-rotor configurations. Several results, showcasing superior performance of the proposed system are provided as well.
\end{abstract}

\section{INTRODUCTION}

The importance of aerial robotics in the modern era is clearly apparent. Unmanned aerial vehicles (UAVs) have shown tremendous promise for a variety of applications, ranging from search and rescue [1], to inspection and construction [2], and entertainment. Accordingly, there continues to be extensive work on design enhancements [3], autonomous flight control [4], and path planning of such aerial vehicles [5].

Among many others, grasping and manipulation are undoubtedly, the two most desirable capabilities that an autonomous robot can possess. In most cases, commercially available generic grasping mechanisms are not suitable for autonomous operation in adversarial environments. The primary reason being their weight and complexity. Thus, custom designed grippers for both ground [6], [7], [8], as well as aerial applications [9] are often favored.

In particular, there has been an ever-growing interest in developing novel technologies, and compliant designs for aerial grasping with UAVs. This enthusism makes sense, since the applications are enormous. According to a recent survey [10], around $86 \%$ of the total Amazon deliveries weigh less than $5 \mathrm{lbs}(2.3 \mathrm{~kg})$, which is well inside the nominal payload range of many commercially available multirotor UAVs. From prestigious robotics competitions [11], to curiosity driven research problems, it is therefore desired

\footnotetext{
*The research reported in this paper was supported by funding from King Abdullah University of Science \& Technology (KAUST).

${ }^{1}$ Usman A. Fiaz is with the Department of Electrical \& Computer Engineering, and the Institute for Systems Research (ISR) at University of Maryland (UMD), College Park, MD, USA. (fiaz@umd.edu)

${ }^{2}$ M. Abdelkader, and Jeff S. Shamma are with the Robotics Intelligent Systems \& Control (RISC) Laboratory at KAUST, Thuwal, KSA. ([mohamed.abdkader| jeff.shamma] @kaust.edu.sa)
}

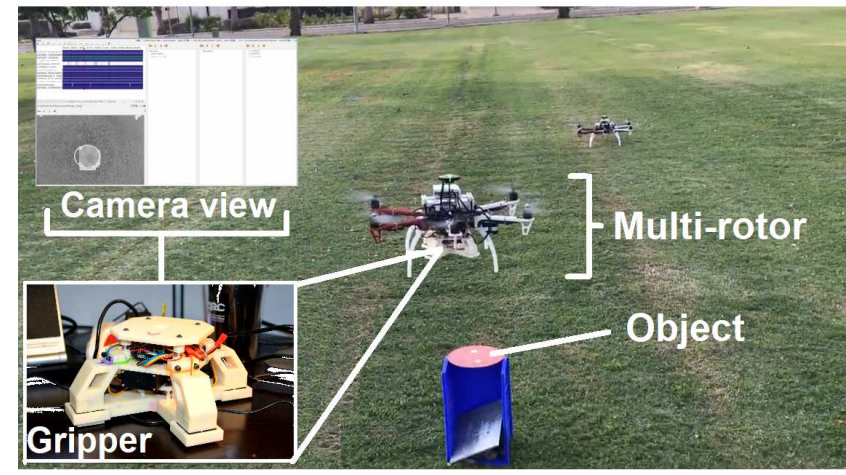

Fig. 1. Our system employs permanent magnets and a novel, dual-impulsive release mechanism, for autonomous aerial transport of ferrous objects in outdoor environments.

for the UAVs, to possess the ability to grasp, and deliver objects of different shapes, and textures, [9] autonomously. For general non-ferrous objects, techniques like self-sealing suction [12], and pinch-hole grasping mechanism [2], have been proposed. They perform well for small objects in structured environments, but in case of heavy, and in particular metallic objects with curvature, they are likely to fail. We intend to address the problem of aerial grasping, subject to reliability of the system, and efficiency of its autonomous operation. In doing so, we limit our focus on the grasping, and transport of ferrous objects only, for reasons specified in [13], [14].

Electro-permanent magnet (EPM) technology has desirable features for ferrous grasping, and thus, has been employed in aerial grasping of magnetic objects in [15]. But it has its own limitations of an active control circuit, and increased system complexity. As is reported in [16], weak magnetic strength, unreliable feedback, and lack of selfassisted alignment, are the biggest challenges with EPM based aerial grasping. Power consumption is another aspect, which makes passive magnets a favorable choice over EPMs.

Hence, we put our focus on developing a novel gripper with permanent magnets, and an impulsive release mechanism. The analysis reveals that the lift provided by our proposed design is enough to support payloads of commercial interest [10], yet our design is much simpler, scalable, and the system complexity is remarkably small. In addition, its performance exceeds EPMs, both in terms of autonomous grasping capabilities, as well as power consumption.

The later sections of the paper are arranged as following. Section II describes the driving force behind this project, as well as a quick comparison with our prior work along similar 
TABLE I

COMPARISON BETWEEN THE TWO PASSIVE GRIPPER DESIGNS

\begin{tabular}{lll}
\hline Parameter & Prior design & Current design \\
\hline Weight $(\mathrm{g}):$ & 155 & 295 \\
Base diameter $(\mathrm{mm}):$ & 100 & 200 \\
Target environment: & Indoor & In/out-door \\
Max. payload (kg): & 1.3 & 2.6 \\
Min. payload (kg): & 0.05 & $<0.01$ \\
Max. sustainable slide (N): & 6.3 & 31.5 \\
Impulsive release: & Single & Dual \\
Feedback sensor: & Infrared (3) & Push-button (4) \\
Camera stabilization: & No & Yes (two modes) \\
Max. no. of objects: & 3 & 4 \\
ROS compatible: & No & Yes (on-board node) \\
\hline
\end{tabular}

lines. In Section III, we describe the model for the system, and introduce the concept of dual impulsive release. Section IV provides a detailed description of the gripper design, and the various hardware components involved in the system. In Section V, we demonstrate the capabilities of the gripper with comprehensive experiments. We also provide a concrete comparison with the EPM technology, before summarizing with a short conclusion in Section VI.

\section{MOTIVATION AND BACKGROUND}

The motivation for this work is twofold. The first comes from our participation in the inaugural version of Mohamed Bin Zayed International Robotics Challenge (MBZIRC) [11]. One of the challenges of the competition required a team of UAVs to autonomously search, locate, pick, and transport several ferrous objects randomly placed in an open field, to a pre-specified drop zone, within a fixed time limit. As is apparent from the challenge description, an integral part of the task was to develop an effective grasping mechanism, capable of autonomous operation in outdoor environment.

The second driving force comes from our previous work [13], where we introduced the concept of passive aerial grasping for ferrous objects. It was found that although the design proposed in [14] performed reasonably well indoors, it is not suitable for outdoor operation. Unreliable sensor feedback, no built-in camera stabilization, sub-optimal magnetic pad arrangement, non-zero minimum drop threshold, and the external torque are some key factors which dictated the need for a new gripper design.

Table I shows a quick comparison with the prior design. Independence from the environment of operation, enhanced sustainable slide, camera stabilization (two modes), ROS compatibility, and most importantly, the dual impulsive release mechanism with counter-torque are the leading improvements in the new design.

It is important to note that our current design is not merely an up-scaled or improved version of the previous one; since that would have only increased the maximum payload range while making the aforementioned issues significant. Hence, almost all the components are designed from scratch, by taking into account the requirements from the challenge, as well as the feedback from prior work. This enabled us in coming up with an intelligent design, which is optimized with respect to all these practical constraints.

\section{Modeling}

This section describes the underlying model for the system. In particular, we introduce the working principle, and the need for the dual impulsive release with counter-torque.

\section{A. Impulsive Release}

The grasping action of permanent magnets is entirely passive and spontaneous, with close vicinity to the object being the only condition imposed. Contrary to the selfintuitive pick-up, the principle behind the dropping of an object is far more interesting, since there is no direct way of deactivating a permanent magnet. We come up with an intelligent mechanism, which utilizes a quick action force to release the object from the magnets grasp. It is based on the idea of impulse of a force. By Newton's second law:

$$
F_{\text {avg }}=m a_{\text {avg }}=m \frac{\delta v}{\delta t}
$$

where $\mathrm{F}_{\mathrm{avg}}$ is the average force acting on the object $(\mathrm{N}), \mathrm{a}_{\mathrm{avg}}$ is the average acceleration $\left(\mathrm{m} / \mathrm{s}^{2}\right), \delta \mathrm{v}$ is the change in its velocity $(\mathrm{m} / \mathrm{s}), \delta \mathrm{t}$ is the time of action of the force (s), and $\mathrm{m}$ is the mass of the object $(\mathrm{kg})$, whose maximum value for a particular magnet in case of a vertical lift, is given as:

$$
m_{\max }=\frac{B^{2} A}{2 \mu_{o} g}
$$

where A is the surface area of the magnet pole $\left(\mathrm{m}^{2}\right)$, B is the magnetic flux density ( $\mathrm{T}), \mathrm{g}$ is the acceleration due to gravity $\left(\mathrm{m} / \mathrm{s}^{2}\right)$, and $\mu_{\mathrm{o}}$ is the permeability of air.

Impulse $\mathbf{J}(\mathrm{Ns})$ is defined as the product of the average applied force $\mathrm{F}_{\mathrm{avg}}$ and its time of action $\delta$ t. Therefore, it can be represented as a change in momentum of the object. Mathematically:

$$
J=F_{\text {avg }} \delta t=m \delta v
$$

Thus, for a given impulse $\mathrm{J}$, the average force $\mathrm{F}_{\mathrm{avg}}$ is inversely proportional to its corresponding time of action $\delta \mathrm{t}$, or in other words, for a given object of mass $\mathrm{m}$, it is directly related to the change in its velocity $\delta \mathrm{v}$.

We exploit this concept in our design to drop the payload attached to permanent magnets. To achieve a desired increase in momentum of the payload (i.e. for a given impulse), one can either decrease the time of action, and effectively increase the applied force or vice-versa. Thus, by using a fast actuator, such as a high speed servo, a sudden push can be produced to undo the force of magnetic attraction between the magnets, and the attached ferrous object, to throw it off the gripper surface.

Consider the simplified model of the release mechanism in Fig. 2(a), where a payload of mass $m$ is attached to the magnets. A drop plate encloses the magnets, which can move upwards or downwards, depending on the servo rotation. Assuming that the plate is rigid, let $\mathrm{F}_{\mathrm{S}}$ be the force it exerts on the object in the event, when servo is activated. Since the pick up does not involve any actuation, the force produced by 


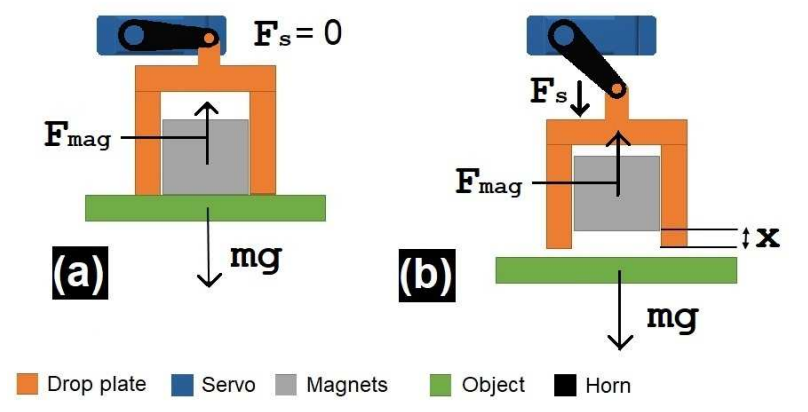

Fig. 2. The conceptual model for impulsive release mechanism. Note that the magnets are entirely static and only the drop plate is mobile. The two states of operation i.e. pick-up (a), and drop (b) are shown separately.

the servo $F_{\mathrm{s}}$ is zero in this state, and the system is stationary at equilibrium. In the drop state, the servo is activated (see Fig. 2(b)). Hence, the drop plate moves down under the influence of $F_{s}$, to overcome the attraction $F_{\text {mag }}$ between the magnets and the object. In doing so, it must travel a vertical distance $\mathrm{x}$, to transfer the requisite momentum (i.e. required impulse) to the object, for its successful separation from the magnets. For the given scenario, the dynamical forces at equilibrium can be expressed as:

$$
m \frac{\delta v}{\delta t}=F_{m a g}-m g-F_{s}
$$

or alternatively as:

$$
F_{s}=\frac{J}{\delta t}-m g+F_{m a g}
$$

Eq. (5) gives the approximate $F_{s}$, i.e. the force, that the actuator must produce for a given impulse $\mathrm{J}$, in order to successfully drop the object. Here, we approximate the magnetic force $F_{\text {mag }}$ as constant, assuming that it is uniform at the air gap. However, in fact, it is a function of the vertical distance $\mathrm{x}$. So, this assumption is only valid when $\mathrm{x}$ is within the air gap limit of the magnetic grip $(<5 \mathrm{~mm}$; this is a design specific parameter). If such is the case, then Eq. (5) holds, and a short time of action $\delta$ t ensures successful drop, with a consequently low requirement for $F_{s}$.

It turns out our assumption is valid on the hardware level as well. There, this shows up more as a compactness constraint on the gripper design. In general, because of the limited space availability on UAVs, the drop plate can only be allowed to move down upto a few $\mathrm{mm}$ for a drop, thus to transfer requisite momentum to an object in that small displacement, the plate must move faster, which is in accordance with the starting assumption.

Another important implication here, which is apparent from Eq. (5) as well, is that the lightest objects are the hardest to drop and vice-versa.

\section{B. External Torque Effect}

In addition to the the forces that we have discussed so far, there is another effect that needs to be accounted for. Using a servo for the actuation of the drop plate leads to an external torque as well. Consider a servo that is firmly attached to a support above, as shown in Fig. 3(a). Its horn is connected
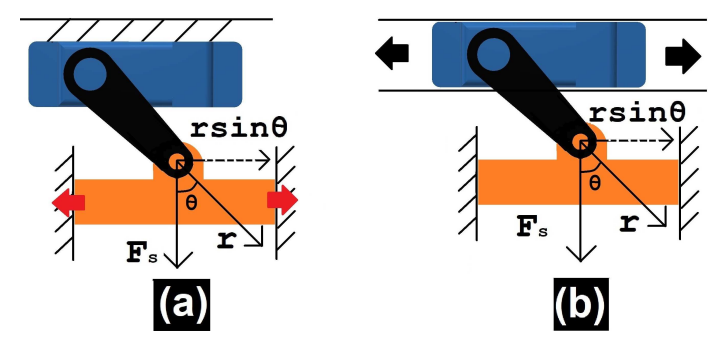

Fig. 3. The external torque effect due to a single servo-actuator.

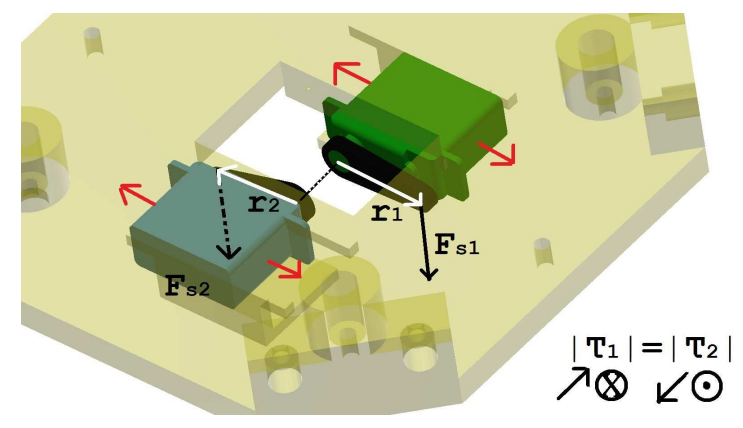

Fig. 4. Dual impulsive release with horizontal translational freedom and counter-torque.

to the drop plate, and controls its motion according to servo rotation. Since our model assumes that the force $F_{s}$ is always vertical, the motion of the drop plate is thus restrained in a vertical column. With this setup, the plate experiences lateral forces along the walls of the sliding support (marked in red). Moreover, there acts an unbalanced, external torque $\tau$ on the system, that results from this single servo-actuated release.

$$
\tau=\mathbf{r} \times \mathbf{F}_{\mathbf{s}}=r F_{S} \sin \theta \hat{\mathbf{n}}
$$

where $\theta$ is the angle between the radius vector $\mathbf{r}$ and applied force $\mathbf{F}_{\mathrm{s}}$, and $\hat{\mathbf{n}}$ is a unit vector into the plane.

Both these factors tend to deteriorate the performance of the release mechanism, by reducing the vertical component of $F_{s}$, which is responsible for the successful drop of payload. Also, this external torque induces a torsional stress on the supports, and increases friction between the sliding components. This accounts for a relatively higher minimum drop threshold in our previous design [14].

\section{Dual Impulsive Release and Counter-Torque}

The problem of lateral forces on the drop plate and supports is solved by allowing the servo a horizontal translational freedom about its mean position, while it rotates to push the drop plate (see Fig. 3(b)). This effectively eliminates any lateral force on the supports, making the vertical component of $F_{S}$ stronger. The issue of external torque is solved by introducing a dual impulsive release, using a pair of identical servos. The axis of rotation is identical for both servos. As shown in Fig. 4, with this setup, the external torque produced by one servo is canceled by an equal, and opposite counter-torque, produced by the other. This synchronized dual-actuation, combined with the horizontal translational freedom of the two servos, guarantees a resultant vertical $\mathrm{F}_{\mathrm{s}}$. 


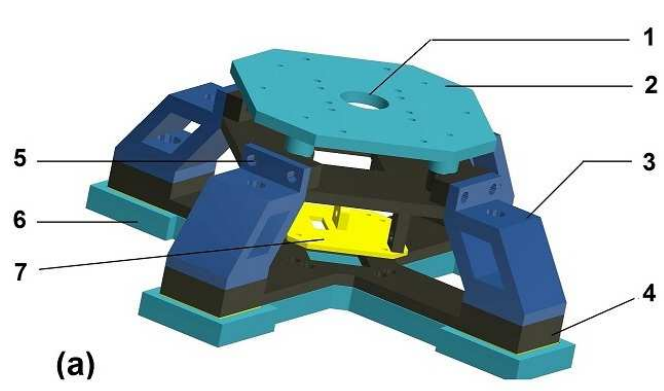

(a)

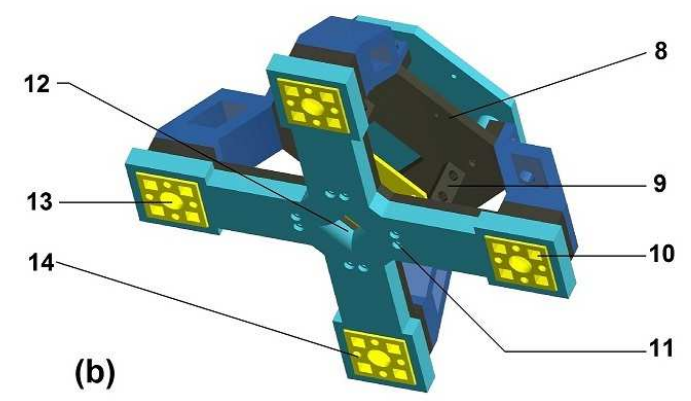

Fig. 5. Intelligent gripper design for aerial transport of magnetic payloads; (a) top side view, and (b) bottom side view; various parts are labeled numerically: (1) cable passage for camera, (2) mount plate, (3) separable support, (4) holding plate, (5) connecting spacer, (6) drop plate, (7) camera plate, (8) servo plate, (9) servo mount, (10) magnet, (11) drop plate support, (12) camera fitting, (13) push button, and (14) flexible magnetic pad.

\section{HARDWARE AND DESIGN}

\section{A. The Gripper}

Fig. 5 shows both top and bottom-side lateral views of the gripper. The design consists of several individual parts assembled together. Each part of the structure is printed with the Stratasys Objet30 3D-printer, using either a rigid, VeroGrey/Black or a flexible, Tango-Black plastic. The base of the gripper comprises of four magnetic pads, each $180 \mathrm{~mm}$ apart and placed at right angles to each other. Thus, it forms a symmetric X-shape which spans over a circle of $200 \mathrm{~mm}$ diameter (see Fig. 5(b)).

Each of the four magnetic pads houses four $\frac{1}{4}$ in cubes of N42 neodymium magnets inside. Thus in total, there are sixteen such magnets, each capable of providing an individual lift of around $10 \mathrm{~N}(1 \mathrm{~kg})$. The placement of these magnets is such that each has the same pole facing down, which unifies their magnetic strength. Unlike [14], the magnets are placed with an insulated plastic separation in between. This is to eradicate any cancellation of the magnetic fields from similar poles in close vicinity. A digital push button is placed at the center of each pad. This serves as a proximity sensor for supplying real-time, pick and drop feedback, as well as the object count, in case of multiple payloads. This simple feedback is found to be reliable irrespective of the lighting conditions, and effective in both indoor and outdoor environments. The drop plate can slide up or down, as controlled by the release mechanism. With this configuration the gripper is capable of grasping up to four ferrous objects (each having a maximum diameter of $90 \mathrm{~mm}$ ) simultaneously.
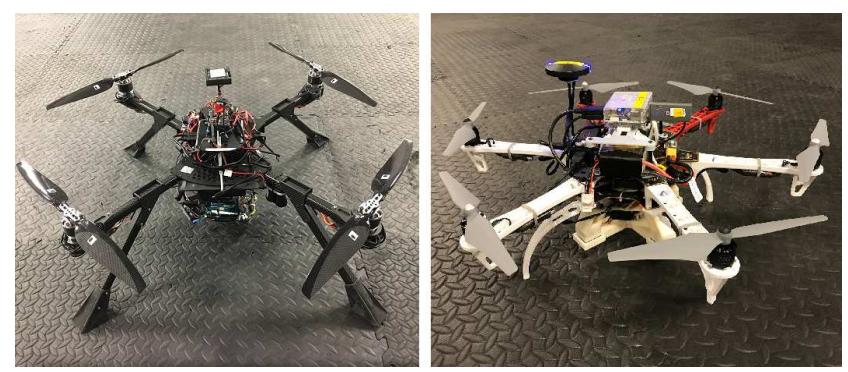

Fig. 6. The UAV platforms used for experiments.

\section{B. Release Mechanism}

As described previously in Section III, the release mechanism consists of two high speed servos (HS-MS7990TH), which are mounted on opposite ends of the servo plate. When the servos are activated, they push the drop plate down with their respective horns. This push occurs in a duration of only 0.15 seconds, and as a result of this dual impulse, the payload is dropped.

\section{Camera Stabilization and Compliance}

We implement two-axis camera stabilization on both local (camera) and global (gripper) levels, in two versions of the gripper with otherwise identical features. Version-I has a fixed camera, and a heavy duty two-axis gimbal is used to mount the whole gripper on to the UAV. In Version-II, we embedded an inherent camera stabilization in the gripper assembly, using a custom designed ultra-nano gimbal.

Due to the permanent nature of magnets, and their spontaneous grasping capability, there is no need to accommodate for any angular misalignment at the local level at individual pads. For Version-I, a global compliance is not required either because of external gimbal stabilization of the whole assembly. In Version-II however, the mount plate is composed of flexible silicone material, which allows it to bend around, up to 15 degrees, in order to tackle any global angular misalignment during the grasping operation.

\section{UAV Platforms}

We used two multi-rotor platforms for experiments, in order to validate our design; a DJI F550 Flamewheel hexarotor, and a customized quadrotor from FalconViz ${ }^{\circledR}$ (see Fig. 6). The payload range of both platforms is around $3.5 \mathrm{~kg}$, and both have an approximate flight time of 15-20 minutes at full payload. The two platforms are equipped with identical electronics and software. A Pixhawk-2 (cube) is used as flight controller and autopilot. An ODROID-XU4 serves as a companion computer, and a high level computational brain for the system. The software is based on ROS, C++, and Python architecture with OpenCV libraries used for simple vision based tracking. The ODROID is responsible for running the vision algorithms for autonomous tracking, and aerial grasping of target objects. Based on this execution, it sends high level position, and velocity commands to the autopilot, which then performs the desired maneuvers. In addition, each UAV is equipped with a number of useful modules and sensors, such as Here+ GPS module, Lidar-Lite 
sensor (range: $40 \mathrm{~m}$ ) for altitude estimation, a Wifi module, and numerous power modules for ODROID, and the servos of the release mechanism.

\section{E. Actuation}

An Arduino Pro Mini serves as a dedicated ROS node for controlling the gripper actuation via USB interface. The actuation and grasping routine for the UAV proceeds in the following sequence. The magnets (being permanent) are activated by default. In the pick-up state, the release mechanism is deactivated. As a UAV detects, tracks, descends, and picks up an object, the feedback signal from the push buttons switches from ' 1 ' to ' 0 '. A ' 1 ' means an object is not picked, while a ' 0 ' means that an object has been picked up successfully. Thus the ' 0 ' message serves as a pick-up confirmation for the main state machine (ODROID). Now, when the UAV reaches the drop zone, the Arduino (ROS node) receives a drop signal from the ODROID (main state machine), and hence it activates the release mechanism. As the object is dropped, the push button feedback switches from ' 0 ' to ' 1 '. Similar to pick up routine, the ' 1 ' message serves as the drop confirmation for the main state machine. Once it receives this confirmation, it proceeds to the next state (i.e. search and pick up) by sending a pick-up signal to the Arduino again, which deactivates the release mechanism once more, and the process continues.

\section{EXPERIMENTS}

\section{A. Maximum Payload}

The maximum payload capability of the gripper is analyzed in two different ways; first for an individual pad, and second, for all four pads used together. For a single pad, the mean value was found to be $7.3 \mathrm{~N}(0.75 \mathrm{~kg})$, and in case of all four pads, we observed a mean maximum payload limit for the gripper to be $25.5 \mathrm{~N}(2.6 \mathrm{~kg}$ ), which is in accordance with our initial design requirement (5 lbs).

\section{B. Minimum Drop Threshold}

As stated in Section III, a minimum drop threshold needs to be defined for the gripper with an impulsive release.The mean minimum drop threshold was observed to be less than $10 \mathrm{~g}$ for the current design. Beyond this payload, the size of the test objects used, got too small for the drop plate to have any affect. So, for all practical objects of considerable size, we can assume the value to be zero. This is a significant improvement compared to [14], where the minimum drop threshold was $50 \mathrm{~g}$ (see Table I).

\section{Maximum Sustainable Slide}

Acrobatic maneuvers can cause the payload to skid-off the gripper surface due to the sliding effect of Coriolis force (see Fig. 7). The situation gets even worse in outdoor environments with windy conditions. Wind increases the lateral drag on the payload, and hence the affect of Coriolis force becomes more significant. Therefore, it is important for the system to have a high sustainable slide, for its reliable operation. We investigated the worst case sustainable slide

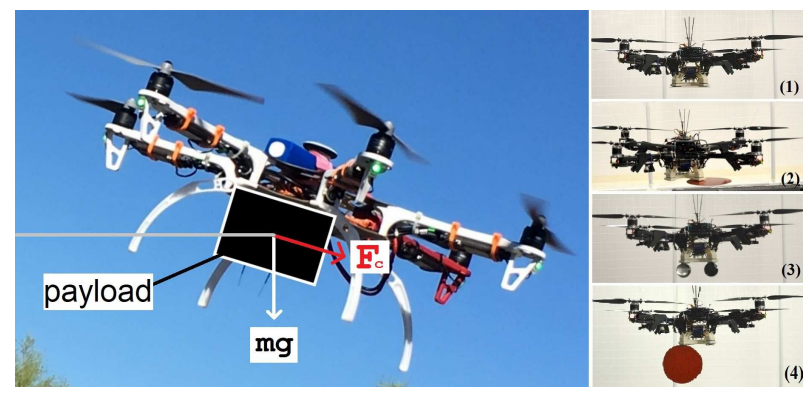

Fig. 7. The Coriolis force $\left(\mathrm{F}_{\mathrm{c}}\right)$ tends to slide the payload off the magnetic grip. The worst case happens when as if the UAV were to perform a 90 degrees roll or pitch maneuver (on left). Demonstration of versatile grasping capability of the gripper, grasping ferrous objects of different shapes and curvature indoors (on right).

for the proposed gripper and observed the values to be 31.5 $\mathrm{N}$ for Version-I, and $27.3 \mathrm{~N}$ for Version-II respectively. This is great, considering that the maximum payload limit for the design is $25.5 \mathrm{~N}$.

\section{Versatility}

We performed a series of grasping tests inside the lab, to show that the gripper is capable of grasping multiple objects of various shapes and curvature. Fig. 7 shows some screenshots from these experiments. The gripper was able to grasp up to four objects of arbitrary shape and form, simultaneously. Test objects included scissors, screw-drivers, ferrous discs, and spray cans. Successful drops for all objects were achieved with a $100 \%$ accuracy as well.

\section{E. Aerial Grasping and Transport}

Finally, we demonstrate the successful operation of the whole system, with a UAV autonomously grasping, and transporting some test objects (weight 350-500 g) in the field.

The objects were placed at random positions, and a drop zone was marked in the field. The state machine for the experiment proceeds as follows. The UAV flies above the field at an altitude of $3 \mathrm{~m}$. Once it detects an object, it tracks, and positions itself over the object. While holding its position, it descends all the way to touch down in a steady fashion, in order to pick up the object. As soon as it gets the confirmation feedback, it starts ascending. After reaching an altitude of $3 \mathrm{~m}$, it heads towards the pre-assigned drop zone, and once there, it drops the object.

We carried out multiple autonomous tests with a number of different objects. Occasionally, we switched to manual flight mode in-flight as well, and performed some aggressive maneuvers with the robot, while it was carrying the payload. None of the objects fell off even in high wind conditions (27 kph). Fig. 8 shows some screenshots from these promising outdoor experiments.

\section{F. Comparison with EPMs}

We conducted a comparative analysis with EPMs in terms of power consumption, as well as payload handling capabilities. An EPM based gripper was developed with three EPM modules (see Fig. 9), so that it had the same surface area as of the proposed design. Extensive experiments under identical 

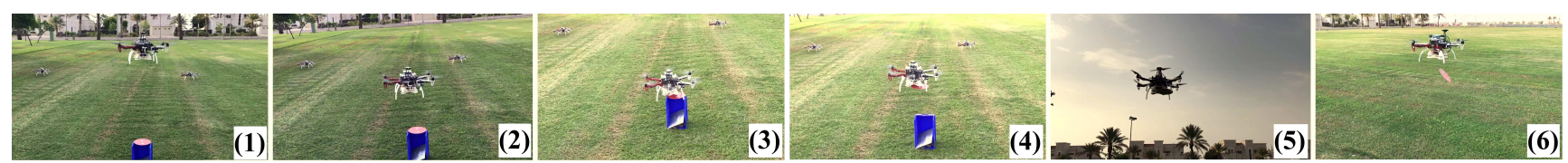

Fig. 8. The hexarotor autonomously grasping the test object in the field: (1) object detection, (2) descent and alignment, (3) touch down and grasping, (4) ascend after pick-up confirmation, (5) fly up to the designated altitude, and proceed to drop zone, and (6) drop at the delivery point.

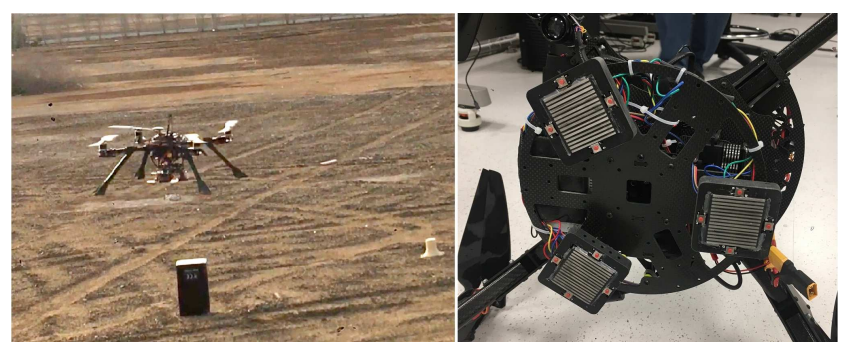

Fig. 9. The quadrotor with an EPM based gripper in aerial grasping action outdoors (left); a close-up of the EPM based gripper with feedback (right).

conditions revealed some interesting features of our design, which outperform EPMs in autonomous aerial grasping. It was observed that an EPM requires perfect alignment, and good contact with the target object in order to grasp it with full power. An in-air activation is not possible, for its magnetic circuit to close, it requires to flush with the object surface. In case of aerial grasping, this requirement is often a big challenge, and it is practically impossible to ensure that the magnets are perfectly aligned to the object on touchdown. This gets even worse in windy conditions, and with objects of irregular shape or curvature. Our design however, is able to grasp the objects spontaneously with no need for special alignment. A total of 30 trials were performed outdoors, under a wind speed of $27 \mathrm{kph}$, and under identical conditions. The success rates for autonomous pick-ups were observed to be $53 \%$ for EPMs, and $97 \%$ for our design respectively. In addition, the study of the current profiles over a complete pick \& drop cycle shows, that the average power consumption of our proposed design $(3.48 \mathrm{~W}$ in total) is much less as compared to the EPMs (14.2 W i.e. 4.75 W per EPM) as well.

\section{CONCLUSION}

We have extended our work on passive aerial grasping, by introducing the concept of dual-impulsive release for ferrous objects. We have described the model for the system, and have emphasized on the need for horizontal translational freedom, and the importance of counter-torque. A new gripper design has been proposed, that does not only realize these concepts, but also out-performs commercially available EPMs in terms of power consumption, as well as autonomous aerial grasping capabilities. However, there remain several open questions too. It remains unclear, that whether or not, this useful concept could be extended to non-ferrous objects in general. Better mathematical models may also be formulated, that would take into account various noise aspects during autonomous aerial grasping.

In future, we are interested in using the developed system for applications such as autonomous package deliveries, aerial perching, docking, and magnetic coupling with the power grid. Micro-UAV deployment and recovery missions with a specifically modified design, autonomous grasping of moving targets, and cooperative aerial transport are also among the future prospects.

\section{SUPPLEMENTARY MATERIAL}

Video link: https://youtu.be/lVjCIHDOfN4

\section{REFERENCES}

[1] A.-a. Agha-mohammadi, N. K. Ure, J. P. How, and J. Vian, "Health aware stochastic planning for persistent package delivery missions using quadrotors," in 2014 IEEE/RSJ International Conference on Intelligent Robots and Systems. IEEE, 2014, pp. 3389-3396.

[2] F. Augugliaro, S. Lupashin, M. Hamer, C. Male, M. Hehn, M. W. Mueller, J. S. Willmann, F. Gramazio, M. Kohler, and R. D’Andrea, "The flight assembled architecture installation: Cooperative construction with flying machines," IEEE Control Systems, vol. 34, no. 4, pp. 46-64, 2014.

[3] J. Thomas, G. Loianno, M. Pope, E. W. Hawkes, M. A. Estrada, H. Jiang, M. R. Cutkosky, and V. Kumar, "Planning and Control of Aggressive Maneuvers for Perching on Inclined and Vertical Surfaces," in IDETC/CIE. Boston: ASME, 2015, pp. 1-10.

[4] H. A. Almurib, P. T. Nathan, and T. N. Kumar, "Control and path planning of quadrotor aerial vehicles for search and rescue," in SICE Annual Conference (SICE), 2011 Proceedings of. IEEE, 2011, pp. 700-705.

[5] M. Abdelkader, H. Jaleel, and J. S. Shamma, "A distributed framework for real time path planning in practical multi-agent systems," IFACPapersOnLine, vol. 50, no. 1, pp. 10 626-10 631, 2017.

[6] M. E. Giannaccini, I. Georgilas, I. Horsfield, B. Peiris, A. Lenz, A. G. Pipe, and S. Dogramadzi, "A variable compliance, soft gripper,' Autonomous Robots, vol. 36, no. 1-2, pp. 93-107, 2014.

[7] P. Xie, O. Ma, L. Zhang, and Z. Zhao, "A bio-inspired uav leg-foot mechanism for landing, grasping, and perching tasks," in Proc. AIAA Atmospheric Flight Mechanics Conference, 2015, p. 1689.

[8] M. Shaqura and J. S. Shamma, "A novel gripper design for multi hand tools grasping under tight clearance constraints and external torque effect," in Mechatronics and Automation (ICMA), 2017 IEEE International Conference on. IEEE, 2017, pp. 840-845.

[9] P. E. Pounds, D. R. Bersak, and A. M. Dollar, "Practical aerial grasping of unstructured objects," in 2011 IEEE Conference on Technologies for Practical Robot Applications. IEEE, 2011, pp. 99-104.

[10] forbes.com, "Turns out amazon, touting drone delivery, does sell lots of products that weigh less than 5 pounds," "https://goo.gl/njWY5j", 2013.

[11] MBZIRC, "Mohamed bin zayed international robotics challenge," "http://www.mbzirc.com/challenge", 2017.

[12] C. C. Kessens, J. Thomas, J. P. Desai, and V. Kumar, "Versatile Aerial Grasping Using Self-Sealing Suction," in IEEE International Conference on Robotics and Automation. Stockholm: IEEE, 2016.

[13] U. A. Fiaz, "Passive magnetic latching mechanisms for robotic applications," Master's thesis, KAUST, 2017.

[14] U. A. Fiaz, N. Toumi, and J. S. Shamma, "Passive aerial grasping of ferrous objects," IFAC-PapersOnLine, vol. 50, no. 1, pp. 10299 10304,2017

[15] A. Gawel, M. Kamel, T. Novkovic, J. Widauer, D. Schindler, B. P. von Altishofen, R. Siegwart, and J. Nieto, "Aerial picking and delivery of magnetic objects with mavs," in Robotics and Automation (ICRA), 2017 IEEE International Conference on. IEEE, 2017, pp. 5746-5752.

[16] R. Bähnemann, M. Pantic, M. Popvić, D. Schindler, M. Tranzatto, M. Kamel, M. Grimm, J. Widauer, R. Siegwart, and J. Nieto, "The ethmav team in the mbz international robotics challenge," arXiv preprint arXiv:1710.08275, 2017 